\title{
Antioxidant Effects of Different Extracts from Root and Aerial Parts of Scorzonera hieraciifolia ${ }^{+}$
}

\author{
Gunes Ak ${ }^{1, *}$, Gokhan Zengin ${ }^{2}$ and Evren Yildiztugay ${ }^{1}$ \\ 1 Department of Biotechnology, Science Faculty, Selcuk University, 42130 Konya, Turkey; \\ eytugay@gmail.com \\ 2 Department of Biology, Science Faculty, Selcuk University, 43130 Konya, Turkey; \\ gokhanzengin@selcuk.edu.tr \\ * Correspondence: akguneselcuk@gmail.com; Tel.: +90-332-232-781 \\ + Presented at the 3rd International conference on Natural Products for Cancer Prevention and Therapy, \\ Kayseri, Turkey, 18-20 December 2019.
}

Published: 26 December 2019

\begin{abstract}
The genus Scorzonera has great potential as traditional drugs and foods in several traditional systems including Turkey. In this current work, we investigated antioxidant properties of different extracts from S. hieraciifolia aerial parts and roots. We used different extracts (dichloromethane, ethyl acetate, hexan, methanol and water). To obtain full picture for antioxidant properties, different methods including radical scavenging (DPPH and ABTS), reducing power (CUPRAC and FRAP), metal chelating and phosphomolybdenum assay. Total phenolic and flavonoid contents were also calculated for each extract. Antioxidant abilities and total bioactive components depended on the solvents used. The highest level of phenolic was determined in methanol extracts, followed by water, ethyl acetate, hexan and ethyl acetate. Similar to phenolic contents, the best antioxidant properties were obtained by methanol and water extracts. Based on our findings. S. hieraciifolia extracts could be valuable source to combat oxidative stress related diseases such as cancer.
\end{abstract}

Keywords: Scorzonera; solvent; reducing power; Turkey

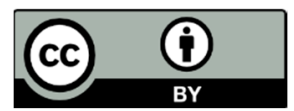

(C) 2019 by the authors. Licensee MDPI, Basel, Switzerland. This article is an open access article distributed under the terms and conditions of the Creative Commons Attribution (CC BY) license (http://creativecommons.org/licenses/by/4.0/). 